\title{
Face Recognition Method for Static Image
}

\author{
Jiang Chunxu, Zhang Xuejiao
}

Baicheng Normal College

Keywords: face recognition; genetic algorithm; static image

\begin{abstract}
The face recognition technology is mainly according to those characteristics that exist large differences among different individuals and are relatively stable for the same person. Which feature forms that will be adopted concretely vary with diverse recognition methods. The static face recognition methods of earlier studies basically included face recognition methods based on correlation matching and geometric features. At present, the face recognition method for static image has the following three major research directions: the first are identification methods based on the connecting mechanism, consisting of elastic graph matching method and general neural network method; the second are the identification methods according to statistics, including the hidden Markov model method and eigenface method; the third are some other comprehensive approaches combining the two.
\end{abstract}

\section{Main methods of face recognition}

Face recognition method based on geometric features

Common geometric features contain face facial features (such as the mouth, nose, eyes, etc.), face shape characteristics and the location features of the five sense organs on the face. When extracting these features, a priori knowledge of the human facial structure is usually applied. In the recognition method according to geometric features, the identification is actually the matching for feature vectors. The judgment method based on Euclidean distance is one of the most commonly used identification methods, and the most critical first step of positive recognition method is suitable normalization. It refers to not depend on human face rotation changes of the image and position scales. Geometry features used in the recognition mainly make geometrical relationships and shape of face organ as based feature vectors, whose components generally indicate the Euclidean distance, angle and curvature of specified two points on the face. For example, Brunelli and Piggio extracted 35D face feature vector method for pattern classification by using integral projection. Face recognition method based on geometric features has the following three advantages: (1) conform to the mechanism of human face recognition, easy to understand; (2)only needing to store a feature vector for each image with a smaller amount of storage; (3)less sensitive to the change of the light. But this method also has the following three shortcomings: (1)it is very difficult to extract stable feature from the image, especially when the feature is covered; (2)it has poor robustness for strong attitude and the expression changes; (3) general geometric characteristics merely describe the basic shape and the structure relationship of the population, while it ignore the local fine features, thus resulting in loss of patial information, so this method is more suitable for rough classification.

Face recognition method based on template matching

Face recognition method based on template matching is a classic method of face recognition, which directly calculates the matching degree between two given images. Therefore, this method requires that two images have the same dimensions, light conditions and orientations, so we have to do the pre-processing work for intensity normalization and scale normalization. In order to overcome the shortcomings of general template matching, the elastic template matching method is proposed. Flexible template defines a set of adjustable parameters designed according to a priori knowledge of the characteristic shape. For purpose of obtaining the value of this set of parameters, a 
priori knowledge of the image edge, valley, peak, intensity information and feature shape should be used to design a suitable energy function. The set of parameters is adjusted toward the direction in which the energy decreases, then the template shape corresponding to this set of parameters best meets the requirements of characteristic shape when the energy reaches the minimum. Brunelli and Poggio has ever compared the recognition method based on geometric features and template matching, and reached the following conclusions: the recognition method based on geometric features has the advantages of a small memory requirements and fast identification speed method, its recognition rate is inferior to that of the template matching recognition method.

Eigenface method

Eigenface method is a face recognition and description techniques exported from the principal component analysis (PCA), which regards the image area in the face as random vector, then adopts $\mathrm{KL}$ conversion to acquire orthogonal KL substrate. Because the substrates corresponding to the larger values have a similar shape to feature face, we call it feature face. We make use of the weighted linear combination of these substrates to describe, express and approximate face image, and with this method we can make face synthesis and recognition. Consequently, the identification process is actually that the face image is mapped to the subspace formed by the characteristic face, and then it is compared with the known face position in the feature face space to identify the human face. Feature face recognition method has the following advantages: (1)better stability and displacement invariance (2) feature vector is proportional to the height of the image (3)transposition invariance. Disadvantages: it is significantly affected by changes of expression, light intensity, angle and perspectives, and has poor robustness. In order to overcome the above drawbacks, the researchers developed many improved methods according it: such as ambiguity analysis method, Gemini space method, Fisher face method and so on.

Hidden Markov model method

Hidden Markov Model is a set of statistical model used to describe the signal statistical properties, mainly used in speech recognition in the earlier stage. HMM applies Markov chain to simulate changes in the statistical properties of the signal, and we indirectly describe this variation by observing sequence, therefore, this process is a double random process. In HMM, the nodes represent the state, and the oriented-edges represent transfer between states. A state may have any of the features in the feature space, while for the same characteristic, the probability of different states showing this feature is different. The merit of HMM is that it allows a greater facial expression change, such as a large rotation of the head, and the defect is the higher complexity of implementation.

\section{Method based on neural network}

A neural network is complex system that models animal nervous systems and makes use of interconnected simple processing units to compose, which is devoted to solve behavior control and complex pattern recognition problems. Neural network method is used to front face recognition and face detection and has achieved good results. Early neural networks for face recognition is auto-associative mapping neural network Kohonen developed, which is used to restore the original intact face when the face of the entered image is polluted seriously by noise or defected partially. Later Cottrell et al take advantage of cascading BP neural network for face recognition, and it has better recognition capability for the portrait with changes of illumination or partially damaged portrait. Whereas Intrator and others adopt a unsupervised / supervised (BCM / BP) hybrid neural network to recognize the face. Neural network approach avoids complex feature extraction work, and you can get implicit expression on face recognition rules and laws through adaptive learning, and it also has very strong robustness and fault tolerance. Furthermore, neural networks deal with information in parallel ways, which can be implemented in hardware and can significantly improve the speed. 


\section{Elastic graph matching method}

Elastic graph matching method is a kind of methods based on the dynamic link architecture (DLA). Its approach is to show human face with sparse graph in trellis, in which use feature vector obtained through Gabor wavelet decomposition of image position to mark the nodes, and use the distance vector connecting nodes to label the edges of diagram. When matching, the first is to search the model diagram most similar with the input image, and then to figure the best match for each node position, by which a deformed plot will be generated, and its node approximates the location of corresponding points in diagrammatic figure. The merit of elastic graph matching method is insensitive to displacement, light, scale changes and rotation. The defect is that it needs to calculate the model diagram for every stored face, and therefore the computation is complex and the storage capacity is large. The above description presents several kinds of classic face recognition methods, and it can be seen through the analysis, each method has its advantages and disadvantages, so some researchers tend to a variety of methods together, or use different types of features at the same time. Face recognition primarily draws on human facial features, namely on the basis of those measures, which have a big difference among various individuals and is relatively stable for the same individual. Because the human face is not rigid, it is more suitable to describe with the elastic model. In consequence, any feature extraction methods based on cut bulk properties are immune to the satisfactory results. Moreover, face recognition is a unique process of human vision, which must combine with the research achievements of human physiology and psychology. Thus the effective combination of various methods will be an inevitable trend for future research, as well as how to combine it with other biometric-based identification systems to improve recognition efficiency is also a very meaningful research direction.

\section{Genetic algorithm (GA) application in face recognition}

Vector quantization technique is an efficient data compression technology developed in the last ten years, now widely used in areas such as image coding. The vector quantization is defined as a set of maps from k-dimensional vector set Q (image block) to a finite set //, whose core is the code book. The capacity of high training set space is set as $\wedge$ rc, and the capacity of codebook space is $C$. For larger Nc and C, it is difficult to quickly search the global optimal classification by means of traditional search algorithm in such a massive category. Wang Jin et made use of the selection mechanism of GA algorithm to adjust the sub error of each region, sequentially put forward an improved competitive learning algorithm-error selection competition learning (DSCL). K. Zeger and E. Yair introduced the stochastic approximation method into vector quantization design, creating a competitive learning (CE) method. Even though this method is simple and can find the global optimal solution, the drawback is the lack of use of the code word, easily leading to local optimal solution. The selection mechanism of the genetic algorithm, however, can well overcome this shortcoming, making it avoid the local optimal solution so as to find global optimal solution. The image restoration is to restore a deteriorative (degraded) image to its original appearance as far as possible.Currently many effective methods were proposed, and these classical methods generally can be divided into statistical analysis methods and deterministic calculation methods. However, most of them need to assume that the deteriorative images not only meet positive constraints, but also to satisfy generalized equilibrium conditions, limiting the effectiveness of their practical applications. By introducing the genetic algorithm, we can well get over these weaknesses. Aiming at the premature convergence problem in genetic algorithm, Xu Lizhong designed a kind of general operator of genetic manipulation, and proposed image genetic recovery method based on the operator, which can solve premature convergence to a non-global optimal recovery image problems in simple genetic algorithm. Fractal image compression method has received increasing researchers' attentions for its potentially higher compression ratio. Since A. Jaequin and M. F. Bamsley had accomplished the first automatic coding scheme, the fractal compression method has been studied and improved by more and more researchers. On basis of the characteristics of fractal and block 
partitioned iterated function system (PIFS), Wu Gengshi and others raised a kind of genetic search algorithm for solving the self-similar block matching with global optimum, which conquer random search and local optimality of classification matching algorithm occurring in the fractal image compression process, and it is a search algorithm with global optimization.

\section{References}

[1] Bledsoe W W. the model method in facial recognition [M].Panoramic Research Inc,Palo Alto, CA,2009

[2] Kannde T Picture processing system by computer complex and recognition of human faces [R].dept of Infornation Science,Kyoto University,1973

[3]Samal A, Iyengar $P$ Automatic recognition and analysis of human faces and facial expressions:Asurvery[J].Pattern Recognition,2008 